\title{
The Fundamental Properties and Application Characteristics of Foam Fluid for Enhanced Oil Recovery
}

\author{
Junhua BAI ${ }^{1, a}$, Zhen WANG ${ }^{1, b}$ \\ ${ }^{1}$ College of Petroleum Engineering, Xi'an Shiyou University, Xi'an, 710065, China \\ aemail: baijunhua0112@sina.com, bemail:wangzhen@163.com
}

Keywords: Foam Fluid; Enhance Oil Recovery; Application in Oilfield

\begin{abstract}
According to the research status of foam fluid, by referring to a mount of literatures, the characteristics and superiority of foam fluid for enhanced oil recovery were analyzed, the foam displacement of reservoir oil mechanism and dynamics characteristics of oil displacement process were summarized. Meanwhile, the applications of the foam fluid on acidification, fracturing and profile control and water plugging were also analyzed, which contains the research achievements about the model built, simulations, field tests and applications in oilfield. Combining with the success cases in oilfield, the paper could supply a research direction for enhanced oil recovery.
\end{abstract}

\section{Introduction}

The foam fluid, because of its material characteristics, is widely applied for the tertiary oil recovery. The foam material has the capacity of seepage and the displacement of reservoir oil, which can decrease the interfacial tension between the oil and water and water-oil mobility ratio, improve the flushing efficiency, and reduce the sweep area of low-permeability layer.

\section{Theory fundamental}

\section{The generation of foam}

The foam fluid is formed by gas-liquid two phases with highly dispersed, which has larger apparent viscosity comparing with active water and gas. The material generates in the porous media, and the generation course can be divided into liquid film hysteresis, necking separation and liquid film breaking.

The common foaming agents applied to oilfield are anionic, non-ionic and ampholytic surfactant. Usually, they are compounded to develop the application. In the process of the forming foam, many factors affect the production and foam dimension, which are surfactant type, molecular structure, pore medium size, surfactant concentration and gas injection rate.

\section{Foam characteristics}

\section{Physical characteristics}

The physical characteristics can be expressed as following[1]:

(1) Compressibility: The foam fluid can be regarded as semi-compressed fluid. Because for the foam fluid, the liquid of it cannot be compressed, while the gas can.

(2) Rheological property: Foam is pseudoplastic fluid, and it has higher apparent viscosity, which will decrease by the increasing shear rate.

(3) Stability: The interface area between the gas and liquid of foam is very large, so the surface free energy is larger, which can keep the foam stable.

\section{Seepage characteristics}

The seepage characteristics in the porous media can be expressed as following [1]:

(1) The foam is bursting and regenerating continuity when in the seepage.

(2) The apparent viscosity of foam in the porous media is very high.

(3) The stability of foam in the oil porous media becomes worse. 


\section{Stability}

The stability of foam flowing in the porous is affected by many factors, not only the same ones as in the balance condition, but also many other factors such as shock, extrusion and friction. Nowadays, the researches of the dynamic condition are focus on experimental data accumulated, theoretical assumptions and building models [2].

\section{Disintegration mechanism}

\section{Burst mechanism}

The foam belongs to unsteady fluid, and the factors which affect the burst are [3]:

(1) The liquid drains in the film: because of the extrusion and gravity between the bubbles, the liquid of foam liquid drains continually, which makes the film thinner, elasticity decreased until burst.

(2) The gas diffusion through the film: According to the Laplace equation, the pressure of small bubble is larger than the big one, so the gas in the small bubble will pass through the film and diffuse to the big bubbles, then the small bubble will become smaller, and the big one become bigger, which can make the foam burst.

\section{Defoaming mechanism in porous medium}

The main reason for bursting bubbles is the effect of suction capillary force which causes the separate pressure. Secondly, the oil can restrain and break the foam, for the diffusion of film drainage and gas occurring between the bubbles.

The research results indicate that the damage degrees to the foam caused by residual oil are different, and the damages to the same foam are also different when it meets different crude oil. The oil is harmful to the generation, extension and block off of the foam, so the oil saturation must decease as possible, then the high temperature foamer could be effective to block off.

\section{Foam displacement of reservoir oil}

The mechanism of displacement of reservoir oil is to take the advantage of the seepage characteristic in porous medium, and the enhanced oil recovery is realized by adjusting the mobility ratio and profile control of foam system $[1,2,4]$.

\section{Dynamic characteristic}

The laboratory tests indicate that when injecting the foam continuously, the orders of product element are the same, which are gas breakthrough, oil zone breakthrough, foam flooding the symbiotic hose and bubble with breakthrough respectively.

\section{Research progress}

There are a mount of researches for the foam displacement of reservoir oil, and mainly focus on building models, numerical simulations and laboratory tests.

The foam displacement model to simulate the oil reservoir condition can be divided into 4 types, the seepage and network model, PBM, fractal flow model and empirical model. Most researches to the numerical model focus on aboard. Like Friedmann developed the formation and burst of foam by PBM, while inland the scholars simulated the 3D conditions by PBM, and get the good simulations to meet the actual situations.

Since the earliest research for the foam to enhance oil recovery in the 1950s by Fried, many contributions have been gotten. The researches of laboratory study and field tests on two phases foam flow got excellent results. By the extension of injection time, because of the existence of alkali, the scaling is formed in the stratum, which may jam the stratum, and the alkali will etch the equipment. On the side, the research results on the effect to the composite foam system indicated that there are also disadvantageous influences, so it is necessary to develop the non-alkali Polymer foam enhanced system. 


\section{Field applications of foam flooding}

Based on the theory, most oil fields made the experiments on the foam flooding. Chai[5] investigated the feasible application to JinZhou Oilfield. ZhaoJun[6,7] made Pilot experiments to QHD32-6 Oilfield by nitrogen foaming agent in 2010, and Chen[2] analyzed the well test results. To solving the problems of the highly fragmented distribution residual oil, water logging, suction uneven and difficulty tapping, two well groups were selected to do the early trials by nitrogen foam flooding in June,2011 for JIN16 [8].

Many countries develop the experiments about the air injection to the light oil reservoir, mainly for the effect of reservoir parameters to the reactivity of crude oil. U.S.A has succeed to the field tests in many fields.[9-14] With the development of the domestic mini air compressor, the air injection technology for enhanced oil recovery improves quickly, the methods of the air-water injected alternately and air foam were used in many fields like BaiSe, ZhongYuan and ShengLi. By the field test results it can be found that there are no safety problems caused by high oxygen content. According to the actual condition of WeiGang field, which has large difference of reservoir physical properties, heterogeneity, and complex underground remaining oil distribution, SunWeifeng[15] found that by the air foam flooding, the profile-log of water injection was improved obviously, start-up pressure raised, water injectivity index drops and the well production effect is evident. The injection of air-water alternately and air foam can block the high permeability zone or large pore path, which can avoid gas channeling. Although the temperature of most oil deposit are all under $70^{\circ} \mathrm{C}$, the output gas oxygen content within the scope of $5 \%$, and there are no security incidents.

\section{Foam acid acidification}

\section{Theory and research status}

For the stratum of low permeability, high heterogeneity, and the foam acid will be selectivity for deep acidification to plug removal, meanwhile the low density residual liquid formed which is helpful for Low pressure cleanup. The chemolysis function of acid liquor will increase the production because it can relieve the formation contaminant, enlarge and connect the reservoir pore, and recover and enhance the permeability of near wellbore area of reservoir.

There are a lot of experiments in domestic and overseas for foam acid acidification. After studying for the corrosion problems of foam acid, Kuibyshev petroleum administration bureau from the Soviet Union considered that the corrosion from foam acid is more powerful than other common acid, the corrosion will strengthen with the higher degree of aeration, and reduce with the concentration of foaming agent. The test result by Wang[14] indicated that the foam acid has the powerful corrosivity, which is acted on foaming agent, corrosion inhibitor and foam stabilizer.

Aiming at the problems of fingering seriously, flow back slowly, and poor acidification caused by the anisotropism and energy shortage for repeat acidizing oil wells of low permeability oilfield, $\mathrm{Hu}[16]$ developed the technology of hard gas foam acid acidification. LiXiaojun[17] researched the stability of the foam acid, and ZhouTiansheng simulated the foam acidizing diversion of horizontal well. By the Stirring method and regarded the half-life period of foam and foaming volume as the main evaluation index, LiYinnian[18] evaluated the annexing agent of foam acid.

\section{Field applications of foam acid acidification}

According to the characteristics of foam acid acidification, Xian and Huang[19] Construct the field test of ZHANG 10-Well in August, 9th,1986. In 2008, Qinghai Oilfield [20] brought in the CPU1200m3/h type nitrogen generating equipment and HY-35/6 foam generator, to make the research and applications of foam acid acidification on shallow oil reservoir. In the field application of JingAn Oilfield, separate layer acid treatment is realized. From construction result, it can be

found that the separate layer acid treatment of process is simple, and the acid discharged completely, for the negative pressure well with obvious contradiction between the layers, the acidification is 
feasible and effect significant.

\section{Foam fracturing}

Foam fracturing is developed since 1970s, which apply to large-scale fracturing, the reservoir of low pressure or low permeability reservoir and water sensitivity, and the production layer is affected by the water-course down layer[21,22,23]. Comparing with the common fracturing, the foam fracturing fluid is superior, which is with higher viscosity, low filtration, low friction, good prop-carrying capacity, smaller reservoir damage and drainage quickly.

For the development process aboard of foam fracturing fluid[24], it can be divided into the stages as following, water-base foam fracturing fluid is regarded as the first generation, the second generator is gelatine foam fracturing fluid, then is crosslinking foam fracturing fluid, the fourth generation is high temperature spumescence foam fracturing fluid. The research in land focus on the non-crosslinked foam fracturing fluid, acid cross-linked CO2 foam fracturing fluid, organoboron cross-linked N2 foam fracturing fluid, delay authigenic heat fracturing, authigenic heat booster class foam fracturing fluid etc. [25].

\section{Foam profile control water plugging}

Many researches are also for the foam profile control water plugging. According to the foam characteristics like shunt, superimposed effect and block off, YangHao[26] analysis the experiment results. DengJun[27] simplified the Sealing channeling model to parallel sand filling tube model, and evaluate the injectivity behavior and plugging. LiZhaomin[28] developed the compatibility of foam and hydrogel microspheres, injection characteristics of multiphase foam system and enhance oil recovery by heterogeneous Cores. To solve the steam channeling problems of Henan heavy oil thermal recovery wells, $\mathrm{Bi}[29]$ carried out the laboratory experiments parameter optimization of nitrogen foam profile control system.

From June 2007 to January 2008, the technicist of Henan Oilfield applied the technology of nitrogen foam profile control[30]. There were field applications ten times in total, and enhanced production of oil 1519.6t, decrease steam channeling to reduce oil 794.7t. In Karamay Oilfield in 2007, the 97 region 28th Well, 970021 Well carried out the experiments of authigenic gas syntactic foam plugging control two times, which are all success [31]. To control the water channeling of high permeability interval and enhance oil recovery, on water flooding wells in Sabei development area, the field test by the technology of nitrogen profile control futile cycle is carried out[32].

\section{Conclusions}

The characteristics and superiority of foam fluid to enhanced oil recovery are organized, and the mechanism of foam displacement of reservoir oil and dynamic characteristics for other oil displacement process is summarized. Meanwhile, the applications of foam fluid to the acidification, fracturing and profile control and water plugging were introduced, which can increase oil production, and a lot of examples were enumerated to prove its successful applications in oil field. All of them will be as theory references for further research of enhanced oil recovery.

\section{References}

[1] Yan Wuhe, Xie Shangxian, Han Peihui. On foam and foam flooding[J]. Oil field chemistry, 1990, 7(4): 380-385.

[2] Chen Zhuanzhuan, Du Shebin. The theory of foam flooding and applications [Z] .Xi'an: Technology research press, 2008.

[3] Xiao Ping. The mechanism research on foam flooding[D]. Shangdong: China University of Petroleum, 2007. 
[4] Chen Kai. The research on foam flooding system to enhance oil recovery[D]. Beijing: China University of Petroleum, 2012.

[5] Chai Junliang. The research and application of nitrogen foam flooding in Jinzhou Oil Field. [J]. Journey of China chemistry trade, 2014, 6(10):54-55.

[6] Zhao Jun, Li Feng, Hu Xue. The applications of nitrogen foaming flooding on offshore oilfield[J]. Applied Science and Technology, 2010, 30(4):33-36.

[7] Deng Yuzhen, Liu Huiqing. The research on injection parameter optimization of nitrogen foam[J]. Journal of Sheng li College China University of Petroleum, 2006, 3(60):88-93.

[8] Zhaodi. The research and applications of nitrogen foam flooding on Jin 16 Xing Oilfield [J]. science and technology. 2013, 6(8):46-47.

[9] A.T.Turta, A.K.Singhal. Field foam applications in enhanced oil recovery projects: seening and design aspects[J]. Journal of Canadian Petroleum Technology.2002, 41(10): 328-337.

[10] Tad W.Patzek. Field applications of steam foam for mobility improvement and profile control[J]. SPE Reservoir Engineering.1996, 11(2): 79-86.

[11] FALODE Olugbenga Adebanjo, UDOFIA, Joseph. Evaluating the application of foam injection as an enhanced oil recovery in unconsolidated sand[J]. Journal of Petroleum and Gas Engineering.2015, 6(2): 22-37.

[12] Li Xuesong, Wang Zhijun, Wang Xi. Studies of Microcosmic Foam Driving Mechanism in Porous Medium [J]. Petroleum drilling technology, 2005, 21(4):1-5.

[13] Chen Gongqi, Wang Xiaohui, Molei. Application of air-foam flooding in extra-low permeability reservoir [J]. Petrochemical industry application, 2013, 32(8):23-26.

[14] Wang Yunjiu. The corrosion of foam acid[N]. Drilling production technology, 2011, 11(32).

[15] Sun Weifeng,Li Xianbin. The application of air-foam flooding [J]. Petroleum geology engineering, 2011, 25(3):85-89.

[16] Hu Zhili, Zhou Yaqing, Chen Xiuli. Acid treating technology in self-productive gas foamed acid [J]. Oil drilling \& production technology, 2010, 32(1):85-88.

[17] Zhou Tiansheng, Li Zhaomin, Wangfei. Numerical simulation on the foam acidizing diversion in horizontal wells[J]. Acta petrolei sinica, 2012, 33(3):448-452.

[18] Li Nianyin, Zhao Wen, Jia Hui. Performance evaluation of foamed acid formula system [J]. Chemical engineering of oil \& gas, 2012, 41(3):311-313.

[19] Xian Sigan, Huang Fuliang. The application of acid technology of foamed acid for Chuandong area [J], Drilling production technology, 1992, 15(4):95-98.

[20] Wu Wenrui, Li Huaijie, Sun Zhilin. Research and application of foam fracturing technology [J]. Petrochemical industry application, 2011, 30(10):24-33.

[21] Xiong Youming. The development Status of foam fracturing for domestic and overseas[J]. Petroleum drilling technology, 1992,15(1):46-55.

[22] Ping Yunfeng. Technical research of $\mathrm{CO}_{2}$ foam fracturing technology[D], Northeast Petroleum University, 2012, 5(10):33-40.

[23] Tian Mingwen, He Xinggui, Zhang Shaobin. The research progress of foam fracturing [J]. Drilling production technology, 2010, 7(21):67-69. 
[24] Li Zhaomin, Zhang Jun, Li Songyan. Current situation and prospect of research and application of clean foam fracturing fluid [J]. Special oil and gas reservoir, 2014, 21(5):1-5.

[25] Li Jing. The research and application on fracturing fluid of clean nitrogen foam [D]. Southwest petroleum University, 2010.

[26] Yang Hao, Chen Wei, Deng Jun. Property study of foam divided-flow[J]. Petroleum drilling technology, 2015, 5(15):12-16.

[27] Deng Jun, Duan Zhiyong, He Juan. Experimental Study on the Mechanism of the Foam Profile Control and Channel Plugging [J]. Journey of Chongqing technology college, 2011, 13(1):104-106.

[28] Li Zhaomin, Li Binfei, Xu Yonghui. Study on the flow-diversion characteristics of foam and its application[J]. Journal of Xi' an Shiyou University, 2007, 22(2):100-106.

[29] Bing Changhui, Zhao Qing, Wang Shulin. Research and application of nitrogen foam profile control for heavy oil thermal recovery [J]. Petroleum geology and engineering, 2008, 22(6): 62-65.

[30] Zhao Xiutai, Wang Jing, Wang Zengbao. Research progress of in-depth profile control technology in China oilfield [J]. Applied chemical industry, 2012, 41(9):1596-1603.

[31] Bill, Walt. A comparison of mixed gas foams with $\mathrm{N}_{2}$ and $\mathrm{CO}_{2}$ foam fracturing fluids on a flow loop viscometer[J].SPE Production and Facilities.1995, 10(3): 197-203.

[32] Wang Binyu, Zhang Jun. Application of technology of profile control with nitrogen in Sabei development unit[J]. Sino-global energy, 2007, 12(4):63-67.

[33] Li Zhaoming, Li Ran, Liu Wei. Application and outlook of foam in oil and gas field development[J]. Oil field chemistry, 2013, 23(1):155-160.

[34] Bernard, Holm. Foam Mobility in Heterogeneous Porous Media.Transport in Porous Media, 2003: 52(1): 17-35.

[35] Thompson, Ganski, Zerhboud, et al. Modelling Foamy Oil Flow in Porous Media.Transport in Porous Media, 1999, 35(2):227-258.

[36] Schramm. A mathematical Study for expanding foam. Journal of Colloid and Interface Science, 2003, 264:237-249.

[37] Patzek, Koinis. Low gas-liquid ratio foam flooding for conventional heavy oil[J]. Petroleum Science, 2011, 8(3):335-344.

[38] Law, Yang, Stone. Physical simulation experiment research of air foam flooding in Da Gang oilfield[J]. Advanced Materials Research.2014, 1073(1076): 2335-2344.

[39] Islam, Faroup Ali. Modeling foam delivery mechanisms in deep vadose-zone remediation using method of characteristics[J]. Journal of Hazardous Materials.2012, 2(43): 37-51.

[40] Vassenden, solheim. A study of the morphologies and inversions of model oilfield dispersions[J]. SPE Production Engineering.1990, 5(3): 265-269.

[41] S.H. Chang. The investigation of threshold pressure gradient of foam flooding in porous media[J]. Petroleum Science and Technology, 2011, 29(23): 2460-2470.

[42] Osterloh, Jante. Novel insight into foam mobility control[J]. SPE Journal.2013, 18(8): 416-427.

[43] P.C.Harris. Modelling of carrot tissue as a fluid-filled foam[J]. Journal of Materials Science.2003, 38(9):1933-1938.

[44] Wen Shili, Xiong Qiyong, Jiang Xiaomin. The research and application of foam profile control 
for autogeneous gases [J]. Technical forum, 2008, 11(45):112-114. 